\title{
Cuantificación de formas larvales de Synthesiomyia nudiseta (Diptera: Muscidae) como un criterio en el análisis del intervalo post mortem
}

\author{
OLGER CALDERÓN-ARGUEDAS*, ADRIANA TROYO*, MAYRA E. SOLANO*
}

\author{
LARVAL QUANTIFICATION OF Synthesiomyia nudiseta (DIPTERA: MUSCIDAE) AS A \\ CRITERION IN ANALISIS OF THE POST-MORTEM INTERVAL \\ IN AN EXPERIMENTAL MODEL
}

The number of larvae of Synthesiomyia nudiseta (Diptera: Muscidae) found in corpses was evaluated as a criterion for the estimation of the post-mortem interval (PMI) in a model with rabbit carcasses. New Zaeland rabbits (3,8 kg) were euthanasied during April, July and October of 2002, and January of 2003, three animals per cycle of observation. The corpses were exposed in a forested environment and samples of muscoid larvae were collected three times a week from each corpse during 80 days. Larvae of $\boldsymbol{S}$. nudiseta were identified and counted during the observation period. The presence of $\boldsymbol{S}$. nudiseta maggots was evident in all the observation cycles. Although the number of collected larvae was greater during the first days of their appearance on the body, there was great variability between each carcass. Although observation of the carcasses began on the second day post-exposition (DPE), in most of cycles, the collection of $\boldsymbol{S}$. nudiseta larvae began between the $7^{\text {th }}$ and $11^{\text {th }}$ DPE and ended at aproximately the $30^{\text {th }}$ DPE. Cycle I (April-June) was an exception, where the recollection of $\boldsymbol{S}$. nudiseta started later $\left(18^{\text {th }} \mathrm{DPE}\right)$ and lasted until the end of the period. These observations suggest that $\boldsymbol{S}$. nudiseta is an important species in the colonization of corpses in the Neotropical Region. Although the great variability in number of larvae collected makes the quantitative interpretation unpractical, the finding of this species at a certain time in forensic investigations may support the estimation of the IPM determined by other criteria.

Key words: Synthesiomyia nudiseta, Muscidae, Cyclorrhapha, forensic entomology, mortem interval.

\section{INTRODUCCIÓN}

Los cadáveres en descomposición constituyen ambientes ecológicos complejos que naturalmente son colonizados por grupos particulares de artrópodos.

La sucesión de especies necrofílicas es un elemento a considerar en el cálculo del intervalo post mortem (IPM) ${ }^{1}$, el cual consiste en la estimación de los tiempos probables máximo y mínimo desde la muerte hasta el descubrimiento del respectivo cadáver ${ }^{2}$.

Según algunos, los cuerpos cursan por varias fases en su proceso de degradación. Estas son la

* Centro de Investigación en Enfermedades Tropicales (CIET). Departamento de Parasitología, Facultad de Microbiología, Universidad de Costa Rica. San José, Costa Rica. E-mail: olgerc@ cariari.ucr.ac.cr 
fase fresca (24 horas), la coagulativa $\left(2^{\circ}\right.$ a $10^{\circ}$ día), la descomposición activa $\left(11^{\circ}\right.$ a $16^{\circ}$ día $)$, la descomposición avanzada $\left(17^{\circ}\right.$ a $42^{\circ}$ día $)$ y finalmente la fase seca (luego del $43^{\circ}$ día $)^{1}$.

En relación con lo anterior se ha demostrado que las moscas califóridas (Diptera: Calliphoridae) representan los primeros insectos en iniciar la oviposición y colonización del cuerpo; período que puede durar desde unos minutos hasta unas cuantas horas luego del deceso ${ }^{3}$. El momento en que ocurre la oviposición de los califóridos suele coincidir con las fases fresca y coagulativa ${ }^{1}$.

Cuando la fase de descomposición activa inicia, otros insectos suelen asociarse con los cadáveres. Dentro de estos se encuentran los múscidos (Diptera: Muscidae) ${ }^{1}$.

Dado que Synthesiomyia nudiseta es un díptero bastante frecuente en la región Región Neotropical ${ }^{4,5}$ y ha sido asociado con cadáveres humanos ${ }^{5}$, el presente trabajo pretende valorar la posibilidad de utilizar el análisis de la recuperación de sus formas larvales como un criterio para estimar el tiempo de muerte y aportar información en el esclarecimiento de las circunstancias de un deceso.

\section{MATERIAL Y MÉTODOS}

Como parte de un estudio en el que se han valorado diversos tópicos de entomología forense en modelos controlados, se evaluó la sucesión recuperación de larvas de $S$. nudiseta en el proceso de degradación de cadáveres. En el presente modelo se realizaron observaciones a partir de carcasas de conejo durante cuatro ciclos de observación que iniciaron en los meses de abril, julio y octubre del 2002 y enero del 2003.

Para cada ciclo, tres conejos de la cepa New Zaeland $(3,8 \mathrm{~kg})$, fueron sometidos a eutanasia mediante dislocación cervical, siguiendo los procedimientos éticos respectivos y posteriormente fueron expuestos a un ambiente selvático constituido por la reserva "Leonel Oviedo", Ciudad Universitaria "Rodrigo Facio", San José, Costa Rica. Los promedios de temperatura, humedad y precipitación para este lugar son $19,3^{\circ} \mathrm{C}, 79,8 \%$ y $19,5 \mathrm{~mm}$ para la estación seca (diciembre-abril) y $20,2^{\circ} \mathrm{C}, 85,1 \%$ y $245,4 \mathrm{~mm}$ para la lluviosa (mayo-noviembre).

Para evitar los efectos de eventuales depredadores, cada conejo se colocó en una jaula de cedazo cuyas dimensiones fueron de 80 × 30 x $25 \mathrm{~cm}$ la cual se sujetó al suelo circundante por medio de ganchos especializados.

Los cadáveres se estudiaron cada tres semanas por ochenta días y con la intención de reproducir la toma de muestras entomológicas en una investigación forense, a partir de cada carcasa se realizó una colecta indiscriminada de las larvas de muscoideos que se encontraban en los principales focos de desarrollo larval. En cada foco se trató de obtener el material de la forma más circunscrita para evitar la perturbación del sistema y se trató de evitar superponer el sitio en las diferentes tomas de muestra sobre el mismo foco. Dicha colecta fue realizada con pinzas entomológicas recuperando cada larva de una en una. El tiempo de colecta fue de cinco minutos en cada uno de los cuerpos. Todos los muestreos se ejecutaron entre las 9 y las 10 horas del día respectivo. Las larvas obtenidas fueron colocadas en viales con alcohol al $70 \%$ y transportadas al laboratorio, donde fueron aclaradas en lactofenol y montadas en medio Hoyer para su posterior identificación, la cual se basó en la clave dicotómica de James (1947) ${ }^{6}$. Los ejemplares que no correspondieron a $S$. nudiseta fueron separados y conservados para estudios posteriores. La variabilidad en los promedios larvales de $S$. nudiseta obtenidos a partir de las colectas diarias en los tres conejos fue evaluada mediante análisis de variancia (ANDEVA) ${ }^{7}, \mathrm{El}$ número de larvas colectadas a partir de los tres cadáveres en cada día fue expresado como promedio de larvas por día utilizando una hoja electrónica de Microsoft Excel $^{\odot}$ y un coeficiente de confiabilidad del 95\%. En los ANDEVA se compararon los promedios del número de larvas de $S$. nudiseta observados desde el primero hasta el último día de su aparición sobre los cadáveres. El período de recolección recolección larval fue considerado como el intervalo comprendido entre el primero y último día del ciclo de observación en que se pudo evidenciar la presencia de larvas de $S$. nudiseta.

\section{RESULTADOS}

La presencia de larvas de $S$. nudiseta fue evidenciada en los cuatro ciclos de observación (Figuras 1 y 2). El número de larvas recuperadas fue mayor en los primeros días de su aparición sobre el cadáver y fue declinando progresivamente en función del tiempo (Tabla 1, Figura 1). A pesar 
Tabla 1. Promedio de larvas de Synthesiomyia nudiseta colectadas por día durante los cuatro ciclos de observación

\begin{tabular}{|c|c|c|c|c|c|c|c|c|}
\hline \multirow[t]{2}{*}{ DPE } & \multirow[b]{2}{*}{$\begin{array}{c}\text { I } \\
\text { Media } \\
\text { DE* }^{*}\end{array}$} & \multirow[b]{2}{*}{$\begin{array}{c}\text { II } \\
\text { Media } \\
\text { DE* }\end{array}$} & \multirow[b]{2}{*}{$\begin{array}{c}\text { III } \\
\text { Media } \\
\text { DE* }^{*}\end{array}$} & \multicolumn{3}{|c|}{ Ciclos de muestreo } & \multirow[b]{2}{*}{$\begin{array}{l}\text { Media } \\
\text { DE* }\end{array}$} & \multirow[b]{2}{*}{$\begin{array}{c}\text { Media } \\
\text { DE* }\end{array}$} \\
\hline & & & & $\begin{array}{c}\text { IV } \\
\text { Media } \\
\text { DE* }^{*}\end{array}$ & $\begin{array}{l}\text { Media } \\
\text { DE* }\end{array}$ & $\begin{array}{l}\text { Media } \\
\text { DE* }\end{array}$ & & \\
\hline 9 & & & 6,0 & 5,6 & & & 16,3 & 27,4 \\
\hline 11 & & & 3,0 & 5,2 & & & 35,7 & 14,3 \\
\hline 14 & & & 2,7 & 4,6 & 16,0 & 17,7 & 42,3 & 6,1 \\
\hline 16 & & & 5,0 & 8,7 & 37,0 & 41,2 & 28,7 & 2,1 \\
\hline 18 & 5,3 & 9,2 & 11,3 & 15,5 & 38,0 & 26,1 & 47,6 & 25,8 \\
\hline 21 & 5,0 & 8,2 & 23,7 & 37,5 & 40,3 & 29,6 & 3,3 & 3,0 \\
\hline 23 & 1,6 & 2,9 & 12,7 & 21,9 & 24,3 & 28,0 & 1,7 & 2,9 \\
\hline 25 & 0,6 & 1,1 & 13,3 & 23,0 & 35,7 & 36,0 & 1,7 & 2,9 \\
\hline 28 & 0,3 & 0,5 & 12,7 & 21,9 & 29,3 & 25,6 & & \\
\hline 30 & 3,6 & 6,3 & 9,3 & 16,2 & 31,7 & 27,8 & & \\
\hline 32 & 1,3 & 2,3 & 8,3 & 14,4 & 27,3 & 30,3 & & \\
\hline 35 & 0,3 & 0,5 & & & 7,0 & 12,1 & & \\
\hline 37 & 0,0 & 0,0 & & & & & & \\
\hline 39 & 0,0 & 0,0 & & & & & & \\
\hline 42 & 0,0 & 0,0 & & & & & & \\
\hline 44 & 0,0 & 0,0 & & & & & & \\
\hline 46 & 2,3 & 4,0 & & & & & & \\
\hline 49 & 1,0 & 1,7 & & & & & & \\
\hline 51 & 0,7 & 0,5 & & & & & & \\
\hline 53 & 0,0 & 0,0 & & & & & & \\
\hline 55 & 2,0 & 3,5 & & & & & & \\
\hline 57 & 5,0 & 8,7 & & & & & & \\
\hline 59 & 2,3 & 4,0 & & & & & & \\
\hline 62 & 0,3 & 0,5 & & & & & & \\
\hline 64 & 0,0 & 0,0 & & & & & & \\
\hline 66 & 0,3 & 0,5 & & & & & & \\
\hline 69 & 0,0 & 0,0 & & & & & & \\
\hline 71 & 0,0 & 0,0 & & & & & & \\
\hline 73 & 0,0 & 0,0 & & & & & & \\
\hline 76 & 0,3 & 0,5 & & & & & & \\
\hline 78 & 1,3 & 2,3 & & & & & & \\
\hline 80 & 2,7 & 4,6 & & & & & & \\
\hline
\end{tabular}

* Desviación Estándar

de que este comportamiento refleja una dinámica poblacional esperada, los datos tuvieron una marcada variabilidad (Tabla 1) que no permite establecer una relación entre el número de larvas colectadas y los DPE que estimarían el IPM.

Aunque el trabajo de observación sobre las carcasas se inició a partir del $2^{\circ}$ DPE, el período de recolección larval de $S$. nudiseta se inició, en la mayoría de los ciclos, entre el $7^{\circ}$ y $11^{\circ}$ DPE (Figura 1, Tabla 1), finalizando aproximadamente en el $30^{\circ}$ DPE. fueron mayores en los primeros días en que apareció el díptero sobre el cadáver (Figura 1), se presentó una gran variabilidad en los resultados por lo que, con la excepción del ciclo IV, no fue posible identificar diferencias estadísticamente significativas entre los tamaños de las poblaciones larvales en función de los DPE del cadáver $(\mathrm{p}<0,05)$ (Tabla 1$)$.

En la mayoría de los ciclos, la recolección de larvas de $\mathrm{S}$. nudiseta se inició entre los primeros 7 y 11 días post exposición (DPE) y concluyó alrededor de los 30 DPE. El Ciclo I (abril-junio) constituyó una excepción en este aspecto. En este ciclo la recolección inició tardíamente ( $\left.18^{\circ} \mathrm{DPE}\right)$ y se prolongó hasta el final del período de observación. 


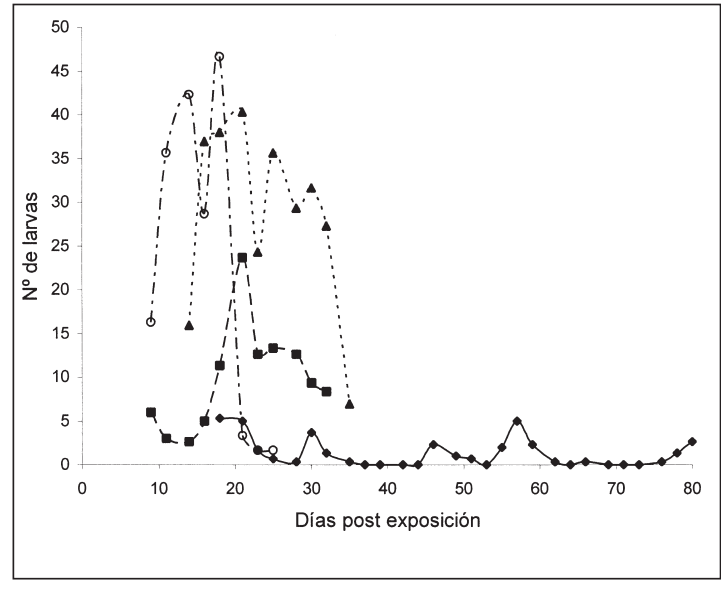

Figura 1. Promedios larvales de $S$. nudiseta en cada uno de los días de observación.

\section{DISCUSIÓN}

Al igual que los califóridos, cuyo papel como indicadores forenses ha sido reconocido ${ }^{8}$, otros grupos de moscas muestran un tropismo definido por la materia orgánica en descomposición. Dentro de estos se encuentran las moscas pertenecientes a la familia Muscidae donde se ubica taxonómicamente $S$. nudiseta ${ }^{l}$. En el presente estudio con base en la recolección y circunscrito al área de ubicación del modelo, se pudo constatar que $S$. nudiseta mantiene densidades poblacionales durante todo el año, razón por la cual la recuperación de larvas tuvo lugar en los cuatro ciclos de muestreo (Figuras 1,2). La recuperación de formass poblaciones larvales, las cuales estuvieron en coexistencia principalmente con califóridos, fóridos y otros múscidos (datos no mostrados), tuvieron mostró la tendencia a ser mayores en los primeros días de su aparición sobre el cadáver disminuyendo paulatinamente en función del tiempo (Figura 1). Este comportamiento podría estar relacionado con el agotamiento poblacional esperado y a la competencia interespecífica por nicho ecológico con otras especies que colonizan cadáveres, sin embargo la gran A pesar de advertir un comportamiento relativamente esperado en lo que respecta a la progresión de un ciclo poblacional, la variabilidad en los resultados no permitió sugiere la posibilidad de establecer relaciones cuantitativas

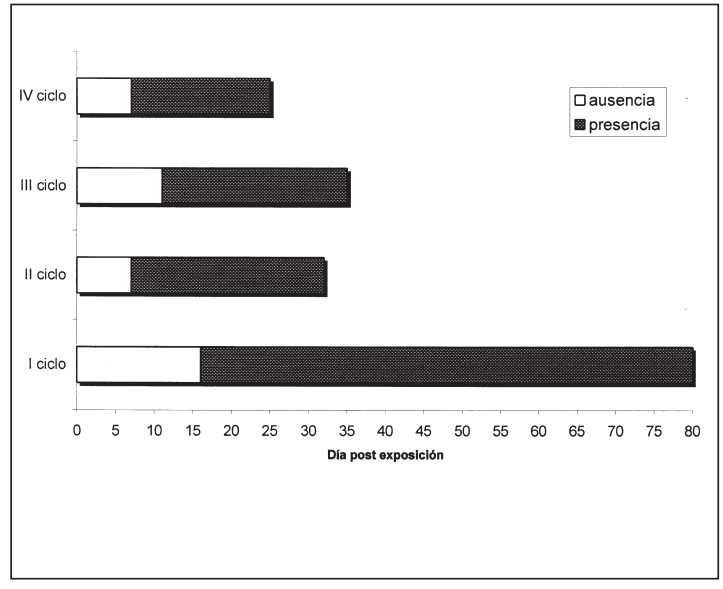

Figura 2. Recuperación larval de Synthesiomyia nudiseta en los cuatro ciclos de observación.

fidedignas entre la colecta larval y el IPM el establecimiento de relaciones entre el tamaño poblacional y los DPE (Figura 1, Tabla 1). Relativo a lo anterior, se debe de tomar en cuenta que diversos factores pueden modular los tamaños poblacionales. En este sentido D'Almeida y colaboradores encontraron una marcada variabilidad en relación con las características y número de oviposiciones que puede realizar $S$. nudiset $a^{4}$. Dichos investigadores, bajo condiciones de laboratorio, pudieron determinar que el número de oviposiciones por hembra fluctuaba entre $1 \mathrm{y}$ 13, mientras que la cantidad de huevos por oviposición iba desde 41,2 hasta 228 . Por otro lado se estima que la tasa de eclosión puede oscilar entre el 20 y el $100 \%{ }^{4}$, por lo que si se relacionan estos aspectos con las poblaciones larvales, es fácil explicar la gran variabilidad observada entre las carcasas (Tabla 1). Aparte de esto, la manera en que se realizó la colecta en la cual se obtuvo una muestra de diferentes poblaciones de muscoideos, pudo haber sido un factor adicional que incrementara la variabilidad entre las poblaciones. En el ciclo IV, se pudieron evidenciar diferencias estadísticamente significativas entre las poblaciones larvales observadas al principio y al final de período de recuperación (Tabla 1 , Figura 1), estas diferencias pueden deberse al comportamiento esperado en una curva poblacional. En relación con la recuperación de larvas, se pudo observar que en todos los ciclos 
ésta tuvo lugar a partir del $7^{\circ} \mathrm{DPE}$, que constituye un momento tardío en comparación con el período de colonización de los califóridos. La recuperación se mantuvo hasta aproximadamente el $30^{\circ} \mathrm{DPE}$, pero una excepción tuvo lugar en el primer ciclo, en el cual la recuperación, aunque mínima se mantuvo hasta el $80^{\circ} \mathrm{DPE}$. En relación con lo anterior se podría hipotetizar que el cambio de estación (seca-lluviosa) pudo incidir sobre estos resultados. Al principio del ciclo tuvieron lugar los procesos esperados de descomposición del cadáver que debido a la baja humedad ambiental, propia de esta época del año, pudieron haber retardado las oviposiciones iniciales de $\mathrm{S}$. nudiseta. En este período hubo un gran consumo de masa muscular debido a la acción de los califóridos y de micro organismos saprófagos. Pero luego de que se empieza a marcar el descenso poblacional esperado, inicia el período lluvioso que aumenta abruptamente la humedad circundante. En esta transición la precipitación cambió de 3,6 mm a principios de abril a 169,3 $\mathrm{mm}$ en el mes de mayo. En estas condiciones la constitución del cadáver pudo cambiar convirtiéndose de nuevo en un ambiente permisivo para las oviposiciones del díptero. En relación con lo anterior, un estudio demostró que $S$. nudiseta, en comparación con otros dípteros calipterados, muestra una gran capacidad para ovipositar en sustratos diversos como pescado, hígado, carne, camarones, heces humanas, calamar y banano. Las tasas de oviposición variaron dependiendo del sustrato, siendo la carne la que permitió las oviposiciones más grandes9. En el contexto del modelo estudiado, los cambios físico-químicos que se generaron en el cadáver por acción de la lluvia pudieron haber permitido oviposiciones constantes sobre remanentes de tejido muscular, piel o pelo, aunque posiblemente el estímulo de ovipostura fue bajo, razón por la cual la recuperación de larvas fue escasa.

En conclusión se puede decir que aunque los la recuperación larvaltamaños poblacionales de $S$. nudiseta no brindan una información completamente confiable en relación con la estimación exacta del IPM útil, se demuestra que esta especie forma parte de la entomofauna asociada a con cadáveres en la región y su presencia podría estar relacionada con un IPM ubicado a partir de la segunda semana del deceso, siempre y cuando el cadáver haya sido expuesto a un ambiente abierto similar al del sitio de estudio. apoyar observaciones tendientes a la determinación de IPM con otras metodologías.

\section{RESUMEN}

El número de larvas de Synthesiomyia nudiseta (Diptera: Muscidae) encontradas in cadáveres fue evaluada como un criterio para la estimación del intervalo post-mortem (IPM) en un modelo con carcasas de conejo. Conejos (New Zaeland) fueron sometidos a eutanasia por dislocación cervical en los meses de abril, julio, octubre de 2002 y enero de 2003, tres animales por ciclo de observación. Los cadáveres fueron expuestos en un ambiente selvático y a partir de cada uno de ellos fueron colectadas muestras de larvas de muscoideos tres veces a la semana durante 80 días. Las larvas de $S$. nudiseta, presentes en la muestra, fueron identificadas y contadas a lo largo del período de observación. La presencia de larvas fue evidente en todos los ciclos. Aunque la observación de las carcasas comenzó en el segundo día post exposición (DPE), en la mayoría de los ciclos, la colecta de larvas de $S$. nudiseta tuvo su inicio entre el $7^{\circ}$ y $11^{\circ}$ DPE y finalizó aproximadamente en el $30^{\circ} \mathrm{DPE}$. El Ciclo I (Abril-Junio) constituyó una excepción ya que la colecta se inició tardíamente $\left(18^{\circ} \mathrm{DPE}\right)$ y terminó al final del período de observación. Las observaciones sugieren que $S$. nudiseta es una especie importante en la colonización de cadáveres en La Región Neotropical. Aunque la gran variabilidad en el número de larvas colectadas hace la interpretación cuantitativa poco práctica, los hallazgos relativos a esta especie en en el contexto de una investigación forense podrían apoyar la estimación del IPM determinado por algún otro criterio.

\section{REFERENCIAS}

1.- ANDERSON G S, VANLAERHOVEN S L. Initial studies on Insect Succession on Carrion in Sothwestern British Columbia. J Forensic Sci 1996; 41: 617-625. 1996.

2.- CATTS E P. Problems in estimating the postmortem interval in death investigations. J Agric Entomol 1992; 4: 245-55.

3.- HALL M, DONOVAN S. Forensic entomology: what can maggots tell us about murders? Biologist 2001; 48: 249-53.

4.- D'ALMEIDA J M, GARCÍA-PIANA M L, TEBALDISELEM C. Comportamento reprodutivo de Synthesiomyia nudiseta (Diptera: Muscidae) sob condiçoes de laboarório. Mem Inst Osw Cruz 1997; 
92: $563-4$.

5.- JIRÓN LF, VARGAS L G, VARGAS-ALVARADOE. Four muscoid flies (Sarcophagidae and Muscidae) associated with human cadavers in Costa Rica. Brenesia 1983; 21: 3-5.

6.- JAMES M T. The flies that cause myiasis in man, USDA, Pub No 631: Washington D. C. 1947. 175 pp.

7.- GREENBERG B. Flies as forensic indicators. J Med Entomol 1991; 28: 565-77.

8.- D'AlMEIDA J M, PINTO DE MELlO R. Comportamento de Dípteros Musocóides frente a substratos de Oviposiçao, em laboratorio, no Rio de
Janeiro, RJ, Brasil. Mem Inst Osw Cruz 1996; 91: 1316.

Agradecimientos: Los autores desean agradecer a Iván Coronado, Ronald Mora, Jacqueline Moya, María Soledad Faba y Adrián Bonilla por su labor operativa ; a Francisco Di Stefano Stéfano Ph. D., Escuela de Biología en la Universidad de Costa Rica (UCR), por su anuencia en la utilización de la Reserva "Leonel Oviedo" y a la Vicerrectoría de Investigación (UCR) por su apoyo financiero al proyecto 803-A2-041.

\section{Carta al Editor}

28 de Mayo de 2005

\section{Doctor}

Hector Alcaíno

EDITOR

Revista Parasitología Latinoamericana

\section{Estimado Señor Editor}

Recientemente fue publicado en la revista el artículo en la modalidad de experiencia clínica "Miasis asociada a síndrome de complejo vascular periférico" de Romero-Cabello y colaboradores (Parasitol Latinoam 59:159-161, 2004). En dicho artículo se vincula a Dermatobia hominis con un cuadro de miasis en una úlcera dérmica.

Diversos aspectos me permiten discrepar de la conclusión que dichos autores hacen en relación con el agente etiológico.

1- D. hominis es un agente causal de miasis primarias, por lo cual sus larvas tienen características biontófagas y su hábitat natural es preferencialmente la piel sana. En el trabajo referido se comenta que la lesión dérmica es muy fétida, con diversas zonas de necrosis y material seropurulento, por lo cual es improbable la asociación entre larvas de $\boldsymbol{D}$. hominis con este ambiente multiplicativo.

2- Las infestaciones dérmicas por $\boldsymbol{D}$. hominis son de tipo forunculoide, usualmente con una larva por lesión, sin embargo en el caso descrito se hace referencia a gran cantidad de larvas infestando la úlcera. En este caso parece probable la ocurrencia de una oviposición o larviposición sobre dicha lesión, situación típica en familias de muscoideos productores de miasis como Calliphoridae o Sarcophagidae, cuyas moscas adultas suelen ser fuertemente atraídas por el material necrótico donde ovipositan o larvipositan. En el caso de D. hominis los huevos son vehiculados por insectos, usualmente hematófagos, cuyas lesiones de picada sirven como entrada para las larvas de la mosca donde inician su período parasitario.

3- Las larvas de D. hominis presentan una morfología típica con un extremo anterior ancho y romo, una cutícula corrugada mostrando espinas laterales prominentes de tonalidad oscura en la región anterior y un extremo caudal aguzado. Estas difieren de la evidencia fotográfica presentada en el artículo, donde las larvas tienen las características de los muscoideos típicos.

Atentamente

Dr. Olger Calderón-Arguedas

Departamento de Parasitología

Facultad de Microbiología

Universidad de Costa Rica

e-mail: olgerc@cariari.ucr.ac.cr 